\title{
Parásitos en expendedores y hortalizas de los mercados públicos. Cuenca 2015
}

\author{
Parasites in sellers and vegetables at public markets. Cuenca \\ 2015 \\ Baculima Tenesaca José Mauricio', Álvarez Serrano Marlene Eliza- \\ beth¹, Zeas Guzmán Ruth Catalina²
}

VOLUMEN 37 | Nº 1 | ABRIL 2019

FECHA DE RECEPCIÓN: 23/11/2018 FECHA DE APROBACIÓN: 10/04/2019 FECHA PUBLICACIÓN: 30/04/2019

1. Universidad de Cuenca 2. Hospital del Río

\section{Artículo Original original Article}

Correspondencia:

mauricio.baculimat@ggmail.com

Dirección:

Ciudadela la Católica, Obispo

Serrano Abad y Miguel León

Código Postal:

010106

Teléfonos:

(07)2341441 - 0992967193

Cuenca - Ecuador

\section{RESUMEN}

Objetivo: determinar la presencia de parásitos en expendedores y hortalizas de los mercados públicos de Cuenca.

Materiales y métodos: es un estudio analítico transversal. La muestra fue de 144 expendedores de hortalizas, realizado en cuatro mercados de Cuenca. Inicialmente se informó a los comerciantes sobre las características de la investigación, se solicitó el respectivo consentimiento informado, se aplicó una encuesta y se les pidió una muestra de heces, posteriormente se compró una lechuga y perejil para su análisis respectivo. A la materia fecal se le realizó un examen coproparasitológico y concentrado de heces mediante el método de Ritchie simplificada, técnica en fresco con solución salina y lugol, para su observación al microscopio; las hortalizas se les colocó por separado en un vaso de precipitado con $400 \mathrm{ml}$ de agua destilada, se dejó 24 horas en reposo, posterior a ello se colocó $8 \mathrm{ml}$ de esta agua en un tubo de ensayo y se centrifugó, el sedimento se observó al microscopio. El procesamiento de muestras cumplió las normas de calidad y bioseguridad. Los datos fueron analizados mediante programa estadístico IBM SPSS Statistics 20 y el programa Epi info 7 (versiones de prueba).

Resultados: se determina presencia de parásitos en los comerciantes en un $68.10 \%$, los principales protozoarios observados son quistes de Entamoeba histolytica $19.03 \%$ y oocitos de Cryptosporidium parvum $16.45 \%$. La contaminación por parásitos en el perejil fue del $44.40 \%$ y en la lechuga el $38.90 \%$. En ambos productos se destaca la presencia de larvas filariformes de Uncinaria spp.

Conclusiones: se observa la presencia de parásitos en expendedores y productos de venta. Los parásitos intestinales más frecuentes son los quistes de Entamoeba coli y oocitos de Criptosporidium parvum. En las hortalizas destaca la presencia de las larvas filariformes de Uncinaria spp.

Palabras clave: parásitos, parasitología de alimentos, muestras de alimentos, verduras, mercados públicos. 


\section{ABSTRAT}

Objective: determine the presence of parasites in sellers and vegetables of the public markets of Cuenca.

Materials and methods: it is a transversal analytical study. The sample consisted of 144 sellers; it was made in four markets in Cuenca. Initially the merchants were informed about the characteristics of the investigation, the informed consent was requested, a survey was applied and a stool sample was requested, later a lettuce and some parsley were purchased for their respective analysis. The faecal matter was subjected to a coproparasitological examination and stool concentrate using the simplified Ritchie method, fresh technique with saline solution and lugol, and for the observation through a microscope; the vegetables were placed separately in a beaker with $400 \mathrm{ml}$ of distilled water for 24 hours, later about $8 \mathrm{ml}$ of this water was placed in a test tube and it was centrifuged, the sediment was observed with microscope. The processing of samples met the standards of quality and biosafety. The data were analyzed using the IBM SPSS Statistics 20 program and the Epi info 7 program (trial versions).

Results: the presence of parasites in sellers is determined by $68.10 \%$, the main protozoa observed are Entamoeba histolytica $19.03 \%$ cysts and $16.45 \%$ Cryptosporidium parvum oocytes. The parasite contamination in parsley was $44.40 \%$ and in lettuce $38.90 \%$. In both products, there is the presence of filariform larvae of Uncinaria spp

Conclusions: the presence of parasites in sellers and products is observed. The most frequent intestinal parasites are the cysts of Entamoeba coli and oocytes of Criptosporidium parvum. In the vegetables, the presence of the filariform larvae of Uncinaria spp is prominent.

Keywords: parasites, food parasitology, food sample, vegetables, specimen handling, public markets.

\section{INTRODUCCIÓN}

La mayoría de los parásitos intestinales son causados por helmintos y protozoarios, generalmente transmitidos por vía fecal - oral, por contaminación del agua y los alimentos; entre esos contaminantes pasivos figuran las verduras que se consumen crudas, mismas que sirven de vehículo a las fases exógenas de los parásitos, como los huevos y las larvas de los helmintos, los quistes y ooquistes de los protozoos intestinales [1-4].

Especial atención se debe dar a los alimentos consumidos crudos, principalmente a las hortalizas, debido al riesgo de contaminación en el medio ambiente y por manipuladores infectados; entre los alimentos que se ingieren crudos, figuran la lechuga y el perejil $[2,5]$.

La contaminación por enteroparásitos en hortalizas comercializadas en los mercados públicos, es un tema que afecta a todos los estratos sociales, por lo que se constituye en un problema de salud pública. Las verduras y hortalizas son un rubro muy importante en la alimentación del ser humano, sin embargo, pueden comportarse como vehículos para la transmisión de protozoarios y/o helmintos intestinales de interés médico [4-6].

Entre los factores determinantes del parasitismo intestinal se consideran como fundamentales a los socioeconómicos y culturales, destacándose las deficientes condiciones de vivienda, falta de acceso a agua potable y saneamiento básico, higiene defectuosa, bajos ingresos, educación deficiente, barreras de acceso a los servicios de salud en general $y$, en especial, a la atención primaria en salud $[7,8]$.

Su impacto es altamente significativo en la población mundial, llegando a ser una de las causas de morbilidad más importantes sobre todo en países en vías de desarrollo [5, 9-11]

Se estima que las infecciones parasitarias, causadas por protozoarios y helmintos, representan el $25 \%$ de las enfermedades infecciosas humanas; una de cada tres personas está infectada por geohelmintos y cerca de 46 millones de niños entre 1 y 14 años están en riesgo de infectarse por estos parásitos, aproximadamente 13 millones de niños en edad pre-escolar ( 1 a 4 años) y 33.3 millones de escolares (de 5 a 14 años) [11,12]. 
Según la OMS, las helmintiasis transmitidas por el suelo afectan a más de 2.000 millones de personas en todo el mundo, los Áscaris lumbricoides afectan a 1.221 millones de personal, Tichuris trichiura a 795 millones, Uncinarias a 740 millones. La infestación se produce por ingestión de huevos presentes en suelos, alimentos o por penetración activa a través de la piel de las larvas presentes en el suelo. Entre los protozoos más frecuentes causantes de diarrea se mencionan a Giardia lamblia, Entamoeba histolytica y Cryptosporidium. Finalmente se considera a la Giardia. duodenalis como el parásito más patógeno [1012].

A nivel local y nacional no se reportan estudios similares, por lo que consideramos que los resultados pueden significar un aporte importante tanto en el conocimiento de la problemática como en el desarrollo de posibles intervenciones.

\section{METODOLOGÍA}

Se trata de un estudio analítico transversal; el Universo estuvo conformado por 228 expendedores de hortalizas en los cuatro mercados de Cuenca. Para el cálculo del tamaño muestral se utilizó la calculadora virtual registrada en PubMed y la Biblioteca Virtual de Salud (Bireme OPS) a partir del cual se obtuvo una muestra de 144 , nivel de confianza del $95 \%$, margen de error del $5 \%$ y una prevalencia del $50 \%$, por desconocer el porcentaje de prevalencia de los factores asociados.

A partir del valor muestral se procedió a la asignación para cada uno de los mercados teniendo en cuenta el número de expendedores y el porcentaje que representan del conjunto de mercados. Con el porcentaje calculado, los sujetos a ser investigados por cada mercado fueron: mercado 1 : 24, mercado 2: 20, mercado 3: 31 y el mercado 4: 69; para la obtención de las hortalizas fue el mismo número de la muestra para la lechuga y perejil. La selección de los comerciantes es no probabilística y por conveniencia; se procedió de esta manera porqué los expendedores no permanecían todos los días en los mercados, a pesar de tener un puesto fijo, se tomó las muestras (heces y hortalizas) a los comerciantes que se les encontraba el día programado para visitar el mercado. El estudio se realizó de enero a diciembre del 2015.
A los participantes se les notificó sobre las características y objetivos del estudio, posteriormente se solicitó el respectivo consentimiento informado, para luego proceder a la aplicación de la encuesta y la solicitud para el procesamiento de una muestra de heces, a cada comerciante se le compró un producto de expendio (lechuga y perejil).

Se incluyeron en el estudio, todos los vendedores que contaban con un puesto fijo en el mercado, mayores de 18 años. Se excluyeron aquellos que voluntariamente manifestaron su deseo de no participar en el estudio.

La muestra de heces se recogió en la mañana, bajo previa indicación y fueron trasladadas inmediatamente al laboratorio para su análisis. Las hortalizas se recolectaron en fundas plásticas herméticas estériles y debidamente identificadas. Con la finalidad de proteger las muestras de contaminación, el manejo de las verduras se hizo con guantes descartables [13]. Las muestras fueron procesadas con celeridad.

\section{Procesamiento de muestras:}

Para el análisis de la materia fecal, se realizaron dos procedimientos parasitológicos: primero, un método de concentrado de heces (técnica simplificada de formol-éter), segundo, un examen coproparasitológico, preparado con lugol el cual es útil para colorear el glucógeno y visualizar los núcleos de los quistes y solución salina al $0.83 \%$ y finalmente preceder a la observación en el microscopio. Tan solo ante la presencia de estructuras similares a parásitos oportunistas, se aplicó la coloración de Ziehl-Neelsen en frío [14-16].

Para el análisis de las hortalizas, se desecharon las hojas manchadas o deterioradas y se usaron las que presentaron aspecto apropiado para el consumo. Se procedió a deshojar las muestras y una vez trozadas se colocaron en un vaso de precipitación con $400 \mathrm{ml}$ de agua destilada. Se dejó en reposo por 12 horas y posteriormente se decantó 9/10 partes de la preparación, el restante se colocó en tubos de ensayo, los cuales fueron centrifugados por 10 minutos a $3000 \mathrm{rpm}$. Una vez descartado el sobrenadante se procedió a obtener la muestra final que fue observada al microscopio con los objetivos de 10X y $40 \mathrm{X}$ $[13,17]$

Para garantizar la validez de los resultados, se realizaron procedimientos de calidad, mismos 
que permiten demostrar la credibilidad y utilidad médica de los datos de laboratorio, los cuales consisten en procesos y técnicas diseñadas para detectar y reducir deficiencias en los exámenes de laboratorio [18].

En el control de calidad en la fase pre-analítica se tomaron en cuenta parámetros como recipiente adecuado, cantidad y calidad de muestra de heces, ya que una muestra escasa afectará la sensibilidad, necesaria para encontrar los elementos que pudiesen estar presentes en ella [19].

En la fase analítica se realizaron distintos puntos de control que permitan comprobar que la metodología es reproducible en el tiempo y con la calidad necesaria. Se realizaron, preparacio- nes dobles de una misma muestra, se volvieron a analizar muestras al azar positivas y negativas, se dispuso de una colección de muestras fecales conservadas y adecuadamente identificadas, que ayuden a la comprobación de parásitos. Se dispuso de fuentes documentales propias, como libros, atlas y de acceso online, para poder efectuar las consultas pertinentes [19-20].

Todos los procedimientos de laboratorio se cumplieron teniendo en cuenta las normas de bioseguridad.

Los datos fueron analizados mediante programa estadístico IBM SPSS Statistics 20 y Epi info 7 en sus versiones de prueba.

\section{RESULTADOS}

\section{Tabla $N^{\circ} 1$}

Presencia de parásitos en 144 expendedores de hortalizas en los cuatro mercados. Cuenca, 2015

\begin{tabular}{lcccccc} 
& \multicolumn{2}{c}{ Positivo } & \multicolumn{2}{c}{ Negativo } & \multicolumn{2}{c}{$\begin{array}{c}\text { Total de muestra } \\
\text { obtenidas }\end{array}$} \\
\cline { 2 - 7 } & $\mathrm{n}$ & $\%$ & $\mathrm{n}$ & $\%$ & $\mathrm{n}$ & $\%$ \\
\hline Mercado 1 & 18 & 12.50 & 6 & 4.20 & 24 & 16.70 \\
\hline Mercado 2 & 12 & 8.30 & 8 & 5.60 & 20 & 13.90 \\
\hline Mercado 3 & 17 & 11.80 & 14 & 9.70 & 31 & 21.50 \\
\hline Mercado 4 & 51 & 35.40 & 18 & 12.50 & 69 & 47.90 \\
\hline Total & 98 & 68.10 & 46 & 31.90 & 144 & 100.00 \\
\hline
\end{tabular}

Elaborado por: los autores. Fuente: base de datos.

Se puede observar que, el $68.10 \%$ de los comerciantes tiene parásitos, sin embargo, al hacer una observación por mercado; de estos quienes trabajan en el mercado 4 muestran el mayor porcentaje: $35.40 \%$ (Tabla No 1). 
Parásitos en expendedores y hortalizas de los mercados públicos. Cuenca 2015 Baculima Tenesaca José Mauricio, Álvarez Serrano Marlene Elizabeth, Zeas Guzmán Ruth Catalina

\section{Tabla $N^{\circ} 2$}

Presencia de parásitos en 144 muestras de perejil y lechuga, en los cuatro mercados. Cuenca, 2015

\begin{tabular}{|c|c|c|c|c|c|c|c|c|}
\hline \multirow{3}{*}{ Mercados } & \multirow{3}{*}{$\begin{array}{l}\text { Muestras } \\
\text { obtenidas }\end{array}$} & \multicolumn{4}{|c|}{$\begin{array}{l}\text { Parásitos } \\
\text { en perejil }\end{array}$} & \multirow{2}{*}{\multicolumn{3}{|c|}{$\begin{array}{l}\text { Parásitos } \\
\text { en lechuga } \\
\text { Positivo }\end{array}$}} \\
\hline & & \multicolumn{2}{|c|}{ Positivo } & \multicolumn{2}{|c|}{ Negativo } & & & \\
\hline & & $n$ & $\%$ & $\mathbf{n}$ & $\%$ & n & $\%$ & $\mathrm{n}$ \\
\hline Mercado 1 & 24 & 15 & 10.40 & 9 & 9.30 & 10 & 6.90 & 14 \\
\hline Mercado 2 & 20 & 6 & 4.20 & 14 & 9.70 & 4 & 4.80 & 16 \\
\hline Mercado 3 & 31 & 17 & 11.80 & 14 & 9.70 & 13 & 9.00 & 18 \\
\hline Mercado 4 & 69 & 26 & 18.10 & 43 & 29.90 & 29 & 20.10 & 40 \\
\hline Total & 144 & 64 & 44.40 & 80 & 55.60 & 56 & 38.90 & 88 \\
\hline
\end{tabular}

Elaborado por: los autores. Fuente: base de datos.

La presencia de parásitos en el perejil, es de un $44.40 \%$ y en la lechuga del $38.90 \%$. En el mercado 4 se registra la mayor presencia de parásitos tanto en perejil (18.10\%) como en la lechuga (20.10\%) (Tabla № 2).

Tabla $N^{\circ} 3$

Tipos de parásitos presentes en hortalizas y expendedores de los cuatro mercados. Cuenca, 2015

\begin{tabular}{|c|c|c|c|c|c|}
\hline \multirow{2}{*}{ Parásitos en hortalizas } & \multicolumn{2}{|c|}{ Total } & \multirow{2}{*}{ Parásitos en expendedores } & \multicolumn{2}{|c|}{ Total } \\
\hline & $\mathrm{n}$ & $\%$ & & $\mathrm{n}$ & $\%$ \\
\hline Quiste Entamoeba histolytica & 12 & 13.79 & Quiste de Endolimax nana & 65 & 28.09 \\
\hline Quiste Entamoeba coli & 19 & 21.83 & Quiste de Entamoeba histolytica & 44 & 19.03 \\
\hline Quiste de Endolimax nana & 36 & 41.37 & Quiste de Entamoeba coli & 40 & 17.04 \\
\hline Quiste Balantidium coli & 4 & 4.14 & Oocito de Cryptosporidium parvum & 38 & 16.45 \\
\hline Trofozoito de Balantidium coli & 2 & 2.29 & Quiste de Enteromonas hominis & 24 & 10.31 \\
\hline Trofozoito de Giardia lamblia & 8 & 9.19 & Quiste de Chilomastix mesnili & 13 & 5.61 \\
\hline Trofozoito de Trichomona hominis & 1 & 1.65 & Quiste de lodamoeba bütschlii & 5 & 2.61 \\
\hline Larva filariforme de Uncinaria spp & 5 & 5.74 & Trofozoito de Trichomonas hominis & 2 & 0.86 \\
\hline Total & 87 & 100 & Total & 231 & 100 \\
\hline
\end{tabular}

Elaborado por: los autores. Fuente: base de datos. 
Los parásitos de relevancia en los expendedores son: quistes de Entamoeba histolytica (28.09\%), oocitos de Cryptosporidium parvum (16.45\%) y trofozoitos de Trichomonas hominis (0.86 \%) (Tabla № 3 ).

Las especies de parásitos más frecuente en las hortalizas fueron los quistes de Endolimax nana

\section{Imagen $N^{\circ} 1$}

Larva de Uncinaria spp, imagen de la izquierda con solución salina y la siguiente con lugol. Lente de 40x

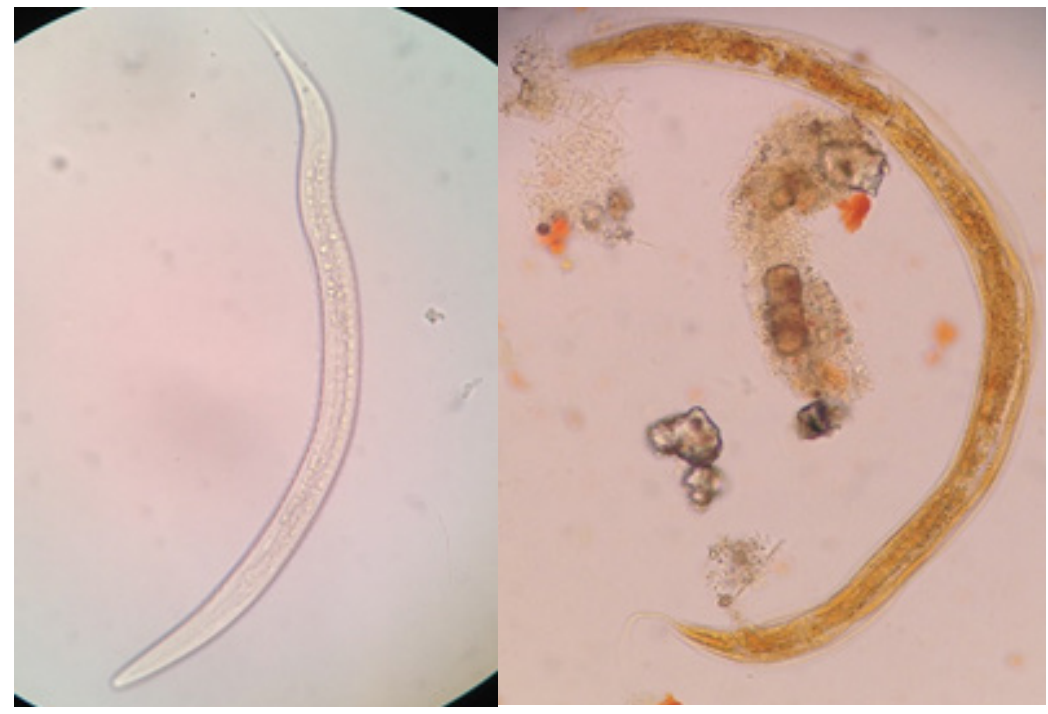

Elaborado por: los autores. Fuente: directa de las muestras de hortalizas.

Tabla $N^{\circ} 4$

Presencia de parásitos según características demográficas. Cuenca 2015

\begin{tabular}{|c|c|c|c|c|c|c|}
\hline & \multicolumn{2}{|c|}{ Positivo } & \multicolumn{2}{|c|}{ Negativo } & \multirow{2}{*}{$\begin{array}{c}\text { RP* }^{*} \\
\text { (IC } 95 \%)\end{array}$} & \multirow{2}{*}{$\begin{array}{c}\text { Valor de } \\
\text { p }\end{array}$} \\
\hline & $n$ & $\%$ & n & $\%$ & & \\
\hline \multicolumn{7}{|l|}{ Edad } \\
\hline 65 o más & 9 & 6.25 & 4 & 2.77 & \multirow{2}{*}{$\begin{array}{c}1.01 \\
(0.69-1.49)\end{array}$} & \multirow{2}{*}{0.924} \\
\hline $18-64$ & 89 & 61.81 & 42 & 29.17 & & \\
\hline \multicolumn{7}{|l|}{ Sexo } \\
\hline Femenino & 96 & 66.70 & 46 & 31.9 & & \\
\hline Masculino & 2 & 1.40 & 0 & 0.0 & & \\
\hline \multicolumn{7}{|l|}{ Residencia } \\
\hline Rural & 36 & 25.00 & 20 & 13.90 & \multirow{2}{*}{$\begin{array}{c}1.11 \\
(0.88-1.40)\end{array}$} & \multirow{2}{*}{0.3644} \\
\hline Urbano & 62 & 43.00 & 26 & 18.10 & & \\
\hline
\end{tabular}

${ }^{*} \mathrm{RP}$ : razón de prevalencia

Elaborado por: los autores. Fuente: base de datos. 
El grupo etario con mayor parasitismo es de 18 a 64 años. Las mujeres son las que mayormente se dedican al expendio de las hortalizas con $98.60 \%$, de ellas un $66.70 \%$ registra parasitismo intestinal. El $61.10 \%$ de expendedores residen en el sector urbano, de los cuales el $43 \%$ está parasitado (Tabla No 4).

\section{Tabla $N^{\circ} 5$}

Presencia de parásitos según características del agua utilizada en el trabajo. Cuenca 2015

\begin{tabular}{lcccccc}
\hline & \multicolumn{2}{c}{ Positivo } & \multicolumn{2}{c}{ Negativo } & $\begin{array}{c}\mathbf{R P}^{*} \\
\text { (IC 95 \%) }\end{array}$ & $\begin{array}{c}\text { Valor } \\
\text { de p }\end{array}$ \\
\hline & $\mathrm{n}$ & $\%$ & $\mathrm{n}$ & $\%$ & & \\
$\begin{array}{l}\text { Obtención del agua } \\
\quad \text { Carro repartidor }\end{array}$ & 31 & 21.50 & 9 & 6.20 & 1.20 & \\
$\quad \begin{array}{l}\text { Red pública } \\
\text { Abastecimiento del }\end{array}$ & 67 & 46.50 & 37 & 25.70 & $(0.96-1.49)$ & 0.1316 \\
$\begin{array}{l}\text { agua } \\
\quad \text { Irregular }\end{array}$ & 31 & 21.50 & 9 & 6.20 & 1,20 & \\
$\quad$\begin{tabular}{l} 
Permanente \\
\hline
\end{tabular} & 67 & 46.50 & 37 & 25.70 & $(0.96-1.49)$ & 0.1316 \\
\hline
\end{tabular}

*RP: razón de prevalencia

Elaborado por: los autores. Fuente: base de datos.

La presencia de parasitismo de acuerdo a la forma de obtener agua por medio de carro repartidor y del abastecimiento de agua de forma irregular, corresponde en los dos casos con un $21.50 \%$ (Tabla $\mathrm{N}^{\circ} 5$ ).

\section{Tabla N6}

Presencia de parásitos según las características, forma de obtención y tratamiento de las hortalizas.

Cuenca 2015

\begin{tabular}{|c|c|c|c|c|c|c|}
\hline \multirow[b]{2}{*}{$\begin{array}{l}\text { Forma de obtención de } \\
\text { hortalizas }\end{array}$} & \multicolumn{2}{|c|}{ Positivo } & \multicolumn{2}{|c|}{ Negativo } & \multirow[t]{2}{*}{$\begin{array}{c}\text { RP* }^{*} \\
(\text { IC } 95 \%)\end{array}$} & \multirow[t]{2}{*}{$\begin{array}{c}\text { Valor de } \\
p\end{array}$} \\
\hline & $\mathrm{n}$ & $\%$ & $\mathrm{n}$ & $\%$ & & \\
\hline Mayorista & 86 & 59.7 & 32 & 22.2 & 1.57 & 0.009 \\
\hline Cultiva & 12 & 8.3 & 14 & 9.7 & $(1.02-2.42)$ & \\
\hline \multicolumn{7}{|l|}{$\begin{array}{l}\text { Lavado de las hortalizas } \\
\text { antes de vender: }\end{array}$} \\
\hline Con agua & 74 & 51.4 & 37 & 25.7 & 0.91 & \multirow{2}{*}{0.333} \\
\hline Ninguno & 24 & 16.7 & 9 & 6.2 & $(0.71-1.17)$ & \\
\hline \multicolumn{7}{|l|}{$\begin{array}{l}\text { Agua utilizada para re- } \\
\text { gar los cultivos }\end{array}$} \\
\hline Agua no potable & 9 & 34.62 & 13 & 50.00 & 0.55 & \multirow[t]{2}{*}{0.2391} \\
\hline Agua potable & 3 & 11.54 & 1 & 3.84 & $(0.26-1.16)$ & \\
\hline \multicolumn{7}{|l|}{ Abono para cultivar } \\
\hline Abono animal & 2 & 7.69 & 3 & 11.53 & 0.84 & \multirow[t]{2}{*}{0.578} \\
\hline Abono orgánico & 10 & 38.47 & 11 & 42.30 & $(0.26-2.68)$ & \\
\hline
\end{tabular}

*RP: razón de prevalencia

Elaborado por: los autores. Fuente: base de datos. 
Se determina que los productos comprados a los mayoristas es un factor de riesgo, registran un mayor índice de parásitos, evidenciándose una asociación y significancia estadística $\mathrm{RP}=1.57$ (1.022.42), $p=0.009$. En este ámbito se determina que, aquellos productos que son "lavados" y han sido cultivados mediante el uso de agua no potable o en su producción fueron "beneficiados" mediante uso de abono orgánico, evidenciaron las más altas cifras de parásitos (Tabla $N^{\circ} 6$ )

\section{DISCUSIÓN}

Se evidencian parásitos en todas las hortalizas de expendio, sobre todo en el perejil con $44.4 \%$, cifra inferior a la registrada en el estudio venezolano de Rivas M. y cols., que encontró un índice de $72.5 \%$ de parásitos, en cambio el estudio realizado en Venezuela por Cazorla D. y cols., obtuvieron un $7.87 \%$ de parásitos en productos similares. En el caso de la lechuga en los mismos estudios, reportó una cifra de parásitos en $20 \%$ en el primer estudio y el otro un $44.4 \%$ de las muestras [21-22], en tanto que nuestro estudio reportó una cifra de $38.9 \%$. La presencia de parásitos en las hortalizas, reflejan condiciones de cultivo, de cosecha y comercialización inadecuadas, desde el punto de vista higiénico sanitario. Los hallazgos demuestran contaminación de los vegetales por heces de origen humano [17, 22].

Los parásitos observados en las muestras de hortalizas fueron: Quistes Entamoeba histolytica (13.79\%), quistes Balantidium coli $(4.14 \%)$ y larvas filariformes de Uncinaria spp (5.74\%), y no patógenos: quistes Entamoeba coli (21.83\%) y quistes de Endolimax nana (41.37\%), y un grupo de parásitos no tan importantes por encontrarse en estado de trofozoito fueron: Balantidium coli, Giardia lamblia y Trichomona hominis, en el estudio realizado por Rivas $\mathrm{M}$. y cols., muestra una similitud en cuanto a las especies de parásitos, más no así en su porcentaje: quiste de Entamoeba histolytica $2.50 \%$, trofozoito Balantidium coli $12.5 \%$, huevo Necator americanus $12.5 \%$, quiste Balantidium coli $2.5 \%$ [21].

Se registra un índice mayor de parásitos intestinales en los comerciantes que obtienen sus productos a los mayoristas con una razón de prevalencia de1.57 (1.02-2.42), $p=0.009$, el estudio de Devera y cols., indica que la mayor presencia de parasitismo, se produce cuando adquieren a comerciantes mayoristas, el mismo se puede deber a las formas de almacenamiento, acopio, transporte, manipula- ción y fiscalización sanitaria, algunos propietarios actuaban como intermediarios en la venta, lo cual puede producir que las hortalizas vaya teniendo más contaminación al manipularse $[2,15,16]$.

En cuanto al uso de agua para el manejo los productos antes del expendio, se determinó que el $46.5 \%$ de los comerciantes parasitados la utiliza proveniente de red pública, en tanto que el $21.5 \%$ se abastece de agua por otros medios, poniendo en más riesgo los que realizan un supuesto lavado de los mismo en balde; al respecto, utilizan agua de riego y manejan sus cultivos mediante uso de abono orgánico, el estudio de Rivas y cols., indican que las condiciones sanitarias de las hortalizas fueron deficientes: los cultivos muchas veces son abonados con estiércol, materia orgánica de origen fecal e irrigado con aguas servidas, así como la mala manipulación en los puntos de venta, al igual que Devera y cols., manifiestan que el mercado donde realizaron el estudio, tiene deficiencias higiénicas en la comercialización de estos vegetales, principalmente en lo que respecta a manipulación, y que además son colocadas al aire libre sin ninguna protección, los vegetales están expuestos a vectores mecánicos [2,21].

\section{CONCLUSIÓN}

El presente estudio evidencia la presencia de parásitos en los productos de expendio, con mayor grado en las muestras de perejil, los principales parásitos observados fueron los quistes Entamoeba histolytica y larvas filariformes de Uncinaria spp

La presencia de parásitos intestinales en los expendedores es elevada, y con mayor intensidad en las mujeres, se destaca la presencia de quistes de Entamoeba histolytica y oocitos de Cryptosporidium parvum.

El factor que predispone al parasitismo en los comerciantes es la adquisición de los productos a mayoristas; otros factores son, los vegetales que son lavados en balde y la obtención de agua en el mercado.

\section{INFORMACIÓN DE LOS AUTORES}

- Baculima Tenesaca José Mauricio. Magister en Microbiología mención Biomédica. Facultad de Ciencias Médicas. Universidad de Cuenca.

ORCID: https://orcid.org/0000-0001-8900-2544 
- Álvarez Serrano Marlene Elizabeth. Magister en Investigación de la Salud. Facultad de Ciencias Médicas. Universidad de Cuenca.

ORCID: https://orcid.org/0000-0002-4726-8050

- Zeas Guzmán Ruth Catalina. Licenciada en Laboratorio Clínico. Hospital del Río.

ORCID: https://orcid.org/0000-0002-7784-2527

\section{CONTRIBUCIÓN DE LOS AUTORES}

B.J: Elaboración de todo el documento, búsqueda de información bibliográfica, toma y procesamientos de las muestras, análisis de la información.

A.M: Revisión y emisión de sugerencias a la información elaborada.

Z.R: participación en el procesamiento de muestras

\section{CONFLICTO DE INTERESES}

Los autores, declaramos que sobre el artículo "Parásitos en expendedores y hortalizas de los mercados públicos. cuenca 2015" no recae ningún tipo de conflicto de intereses.

\section{FUENTES DE FINANCIAMIENTO}

\section{Autofinanciado}

\section{REFERENCIAS BIBLIOGRÁFICAS}

1. Pérez-Cordón G, Rosales MJ, Valdez RA, Vargas-Vásquez $F$, Cordova O. Detección de parásitos intestinales en agua y alimentos de Trujillo, Perú. Rev Peru Med Exp Salud Publica. enero de 2008;25(1):144-8.

2. Devera R, Blanco Y, González H, García L. Parásitos intestinales en lechugas comercializadas en mercados populares y supermercados de Ciudad Bolívar, Estado Bolívar, Venezuela. Rev Soc Venez Microbiol. 2006;26(2):100-7.

3. Cerón D. Identificación de indicadores entéricos en cilantro (Coriandrum sativum) $Y$ PEREJIL (Petroselinum sativum) que se expenden en mercados populares del norte de la ciudad de Quito. Disponible en: $\quad$ http://repositorio.ute.edu.ec/ bitstream/123456789/5102/1/57951_1.pdf
4. Marcano Y, Suárez B, González M, Gallego L, Hernández T, Naranjo M. Caracterización epidemiológica de parasitosis intestinales en la comunidad 18 de Mayo, Santa Rita, estado Aragua, Venezuela, 2012. Bol Malariol Salud Ambient. diciembre de 2013;53(2):135-45.

5. Nicholls S. Parasitismo intestinal y su relación con el saneamiento ambiental y las condiciones sociales en Latinoamérica y el Caribe. Biomédica. diciembre de 2016;36(4):496-7.

6. Brito Núñez JD, Landaeta Mejías JA, Chávez Contreras AN, Gastiaburú Castillo PK, Blanco Martínez YY. Prevalencia de parasitosis intestinales en la comunidad rural apostadero, municipio sotillo, estado monagas, Venezuela. Rev Científica Cienc Médica. 2017;20(2):7-14.

7. Oña- Cisneros F, Garcia D, Costta M, Ruano AL. Prevalencia de parásitos intestinales y comparación de dos métodos diagnósticos en heces de niños escolares de tres parroquias del Distrito Metropolitano de Quito, provincia de Pichincha, Ecuador. Disponible en: https://www. researchgate.net/publication/304580298_ Prevalencia_de_parasitos_intestinales_y_ comparacion_de_dos_metodos_diagnosticos_ en_heces_de_ninos_escolares_de_tres_ parroquias_del_Distrito_Metropolitano_de_ Quito_provincia_de_Pichincha_Ecuador

8. Londoño AL, Mejía S, Gómez-Marín JE. Prevalencia y factores de riesgo asociados a parasitismo intestinal en preescolares de zona urbana en Calarcá, Colombia. Rev Salud Pública. 2009;11(1):72-81.

9. Bracciaforte R, Díaz MF, Vottero Pivetta $\mathrm{V}$, Burstein V, Varengo $\mathrm{H}$, Orsilles MÁ. Enteroparásitos en niños y adolescentes de una comuna periurbana de la provincia de Córdoba. Acta Bioquímica Clínica Latinoam. 2010;44(3):353-358.

10. Cardozo G, Samudio M. Factores predisponentes y consecuencias de la parasitosis intestinal en escolares paraguayos. Pediatría Asunción. 30 de agosto de 2017;44(2):117-25. 
11. Malagón-Londoño G, Moncayo Medina Á. Salud pública: perspectivas. Segunda edición. Bogotá [etc.: Editorial Médica Internacional; 2011.

12. OMS | La Organización Mundial de la Salud y un conjunto de colaboradores dan a conocer un nuevo plan coordinado para tratar a millones de personas que sufren enfermedades tropicales desatendidas [Internet]. WHO. [citado 31 de marzo de 2019]. Disponible en: https://www.who.int/ mediacentre/news/releases/2006/pr60/es/ index1.html

13. Rea MJ, Fleitas A, Borda CE. Existencia de parásitos intestinales en hortalizas que se comercializan en la ciudad de Corrientes, Argentina. Corrientes Univ Nac Nordeste. Disponible en: http://www.iaea.unne. edu.ar/unnevieja/Web/cyt/com2004/3Medicina/M-102.pdf

14. Ash AL, Orihel OT. Atlas de parasitología humana. 5ta edición. Buenos Aires; Madrid: Editorial Médica Panamericana; 2010.

15. Selva DMC, Leytón MRE, Barrera KYA, Téllez A. Frecuencia de parásitos intestinales en expendedores de alimentos ubicados en los recintos de la UNAN-León. Universitas Disponible en: http://www.unanleon.edu.ni/ universitas/pdf/volumen2_nro2/arto4.pdf

16. Vasquez I, Restrepo M, Botero D. Cryptosporidiosis [Internet]. [citado $10 \mathrm{de}$ septiembre de 2015]. Disponible en: http:// www.revistabiomedica.org/index.php/ biomedica/article/viewFile/1916/1941

17. Castillo NAC, Campuzano S. Estudio piloto de detección de parásitos en frutas $Y$ hortalizas expendidas en los mercados públicos y privados de la ciudad de Bogotá DC Nova, enero-junio, año/vol. 4, número 005 Universidad Colegio Mayor de Cundinamarca. Disponible en: http://www. unicolmayor.edu.co/invest_nova/NOVA/ ARTORIG7_5.pdf

18. Pinto P, Ramírez S, Zambrana M. Elaboración del manual de procedimientos para el área de parasitología del laboratorio central del
Hospital del Niño «Dr. Ovidio Aliaga Uría», durante la gestión 2005. [Internet]. 2005 [citado 20 de mayo de 2014]. Disponible en: $\quad$ http://200.7.160.67:8080/rddu/ bitstream/123456789/3687/1/TD573\%20 PINTO\%20SURCO.pdf

19. Jercic M, Oyarce A. Recomendaciones para el control de calidad aplicado al examen parasitológico seriado de deposiciones (EPSD) [Internet]. Disponible en: http://www.ispch.cl/sites/default/files/ Recomendaciones $\% 20$ para $\% 20$ el $\% 20$ Control\%20de\%20Calidad\%20EPSD.pdf

20. Cardona CG. CCI-SEIMC-00 Recomendaciones Para El Control De Calidad Interno: Justificación y Objetivos. [citado 14 de diciembre de 2015]; Disponible en: http:// www.seimc.org/contenidos/gruposdeestudio/ gegmic/dcientificos/documentos/gegmic_ dyc1_2004.pdf

21. Rivas M, Venales M, Belloso G. Contaminación por enteroparásitos en tres hortalizas frescas expendidas en el Mercado Municipal de Los Bloques de Maturín, Monagas, Venezuela [Internet]. Revista Venezolana de Ciencia y Tecnología de Alimentos. [citado 27 de febrero de 2014]. Disponible en: http://www.rvcta. org/Publicaciones/Vol3Num1/ArchivosV3N1/ Rivas_Magalys_et_al._RVCTA-V3N1.pdf

22. Cazorla D, Morales P, Chirinos M, Acosta ME. Evaluación parasitológica de hortalizas comercializadas en Coro, estado Falcón, Venezuela. Bol Malariol Salud Ambient. 2009;49(1):117-125. 